\title{
Ricardo Valderrama Fernández, etnógrafo de las sociedades quechuas del sur andino peruano (1945-2020)
}

\section{Guillermo Salas Carreño}

(iD) https://orcid.org/0000-0003-4770-4550

Pontificia Universidad Católica del Perú

guillermo.salas@pucp.pe

El 30 de agosto de 2020 falleció el doctor Ricardo Valderrama Fernández luego de largos días de lucha contra la Covid-19. Luego de asumir la alcaldía provincial del Cusco a fines de 2019, el doctor Valderrama priorizó su responsabilidad como autoridad liderando los esfuerzos por minimizar el impacto de la pandemia, pese a ser una persona vulnerable a sus 75 años. Nos deja no solo el ejemplo de su compromiso profundo con el bienestar de sus conciudadanos en su rol de autoridad municipal, sino también una larga y fecunda obra antropológica compartida a lo largo de décadas con la doctora Carmen Escalante Gutiérrez, su esposa. Es necesario subrayar que casi todas las publicaciones de Ricardo son en coautoría con Carmen. En este sentido, hago mías las palabras de Lucho Nieto (2020): «Rendir, pues, homenaje a Ricardo es hacerlo al mismo tiempo a Carmen y, en estos momentos, es también una forma de acompañarla en su dolor y pesar».

Egresado y profesor de Antropología en la Universidad Nacional de San Antonio Abad del Cusco, Ricardo ha contribuido notablemente tanto a la disciplina como al conocimiento de las sociedades andinas por sus publicaciones, por su labor docente y por construir activamente un nodo de relaciones entre investigadores locales y andinistas extranjeros investigando o visitando Cusco. A raíz de su deceso se ha comentado extensamente su obra más conocida, el libro Gregorio Condori Mamani. Autobiografía, publicado por el Centro Bartolomé de las Casas en 1977 (Valderrama y Escalante, 1977), reeditado varias veces y traducido también al inglés, noruego, alemán y holandés (por ejemplo, Valderrama y Escalante, 1996; Zevallos Aguilar, 1998, p. 241). Con el riesgo de repetir algunas 
cosas que ya se han dicho, me detendré por un momento en este libro. Junto con Kay Pacha, que reúne las narraciones de Bernabé Condori con Rosalind Gow (Gow y Condori, 1976), publicado un año antes también por el Centro Bartolomé de las Casas, Gregorio Condori Mamani. Autobiografía inaugura un formato de publicación bilingüe que privilegia la narración en quechua en si misma acompañada de su traducción castellana en la página siguiente. Este formato es importante, pues provee un corpus de narrativa conversacional en quechua - inevitablemente editada y transformada al ser transcrita-acompañada por una traducción al castellano, haciendo posible una comparación párrafo por párrafo. El texto en quechua, la traducción al castellano y la estructura formal de la compaginación ha hecho de este libro un clásico de uso particularmente frecuente por una gran cantidad de estudiantes del quechua en aulas. En él hemos encontrado una gran herramienta de aprendizaje, siempre retadora y fascinante. Este formato ha sido, pues, sumamente productivo como parte importante de múltiples esfuerzos por desarrollar el corpus literario quechua contemporáneo que apuntale procesos de desarrollo de literacidades quechua, así como brindar insumos indispensables para el aprendizaje del quechua como segunda lengua, aspectos todos asociados a combatir la condición del quechua como idioma oprimido ${ }^{1}$.

Es claro que su lúcida apuesta por este tipo de publicación bilingüe ha sido firme y sostenida. Similar formato encontramos en sus obras posteriores, como Nosotros los humanos. Nuqanchik runakuna: Testimonio de los quechuas del siglo XX (Valderrama y Escalante, 1992) o La doncella sacrificada: mitos del valle del Colca (Valderrama y Escalante, 1997a).

Más allá de su estructura formal Gregorio Condori Mamani. Autobiografia es el testimonio tanto de Gregorio como de su esposa, Asunta Quispe. En ellos están plasmadas duras historias de migración, pobreza, abuso y violencia que han moldeado las vidas de los más excluidos en la sociedad regional. Tan temprano como en la década de 1970, estos testimonios nos muestran una sociedad regional caracterizada por la migración estacional y la movilidad entre espacios rurales y urbanos, al tiempo que una visión quechua de los principales procesos del siglo $\mathrm{XX}$, donde surgen una peste que diezma comunidades rurales y el desamparo infantil, la cárcel arbitraria y las disputas del apu Ausangate con el gobierno, las luchas de Hugo Blanco y las cuevas de los gentiles, la violencia de género y la ternura en la precariedad.

Sobre la noción de idioma oprimido, ver Albó (1973). 
La complejidad de las narrativas del libro dio pie desde un inicio a múltiples lecturas y debates desde su publicación. Un ejemplo temprano fue el debate sobre la vigencia, vitalidad y relevancia del conocimiento quechua entre Jürgen Golte (1980, 1981), Juan Ossio (1981) y Henrique Urbano (1981).

Similar complejidad aplica para Nosotros los humanos. Ñuqanchik runakuna (Valderrama y Escalante, 1992), aunque en lugar de estar articulado por los diálogos con una pareja mayor, lo están por aquellos con Victoriano Tarapaku, líder con larga experiencia de servicio a su comunidad, y Lusiku Ankalli, un renombrado abigeo, ambos miembros de una comunidad campesina de la zona de Cotabambas, Apurímac. Contrariamente a lo que uno podría esperar, las narraciones del abigeo, lejos de elaborar sobre episodios violentos, giran alrededor de aventuras más bien amorosas. La textura misma de las narraciones en estos libros muestra claramente la profundidad e intimidad de los diálogos en los que se fundan y que solo pueden emerger a través de relaciones de confianza y respeto tejidas pacientemente. Un artículo previo, pero claramente relacionado con este libro, se centra más claramente en los testimonios de abigeos: «Nuestras vidas»: abigeos en Cotabambas (Valderrama y Escalante, 1990). Otro artículo no muy conocido emergió en Huancavelica y es pionero en el registro de testimonios de la época de la hacienda en una región en la que no se había llevado adelante mucho trabajo etnográfico: Testimonio de un pongo (Valderrama y Escalante, 1988c).

Un corpus muy relacionado - pero relativamente diferente- de su trabajo consiste en el registro de lo que usualmente se denomina «mitología andina», esto es, conversaciones sobre seres y eventos clasificados como sobrenaturales en la sociedad dominante. Nuevamente Ricardo y Carmen brindan versiones bilingües de estas narraciones, proporcionando un registro excepcional de estos mundos quechuas contemporáneos. Estos se encuentran en artículos como El apu Ausangate en la narrativa popular (Valderrama y Escalante, 1975), Mitos y leyendas de los quechuas del sur del Perú (Apurímac, Cusco) (Valderrama y Escalante, 1978), El inca vive (Valderrama y Escalante, 1995), Agua, riego, alianza y competencia en mitos del Cusco (Valderrama y Escalante, 2000), El inca en la tradición quechua contemporánea (Valderrama, 2015). El corpus de este tipo de narraciones más extenso que emergió en un solo contexto etnográfico se puede encontrar en el libro La doncella sacrificada: mitos del valle del Colca (Valderrama y Escalante, 1997a). De forma similar a los trabajos más bien centrados en testimonios — que inevitablemente incluyen también narrativas mitológicas - , estos textos ponen el mayor esfuerzo en registrar y publicar las narrativas mismas antes que focalizarse en algún tipo de interpretación. Esta es una apuesta consistente en toda su obra 
y no es un menor posicionamiento político en el que ponen en primer plano las voces de sus interlocutores. Si alguna crítica podría hacerse a este formato es que, en gran medida, están ausentes de los textos impresos las huellas dialógicas de la emergencia y el contexto de estos relatos. Por privilegiar las voces de los interlocutores se borran las huellas del diálogo con los etnógrafos que inevitablemente también forman parte fundamental de estas narrativas, tanto como el contexto concreto de emergencia narrativa (ver Mannheim y Van Vleet, 1998).

Un tercer grupo de trabajos que usualmente también incluyen testimonios y narraciones está más bien centrado en descripciones de prácticas quechuas. En ellos, Ricardo y Carmen muestran una agudeza y finura etnográfica notable, sobre todo en la descripción de prácticas rituales. Entre estos trabajos se pueden mencionar el artículo Sistemas de riego y organización social en el valle del Colca: caso Yanque (Valderrama y Escalante, 1986) y el libro Del Tata Mallku a la Mama Pacha: riego, sociedad y ritos en los Andes peruanos (Valderrama y Escalante, 1988a). Ambos trabajos están dedicados a la descripción etnográfica de las prácticas alrededor del riego y cómo estas están relacionadas con la estructura social en comunidades del valle del Colca, Arequipa. Entre otros textos dedicados a la descripción etnográfica también encontramos los siguientes artículos, todos situados en la zona de Cotabambas: Pacha T'inka o la T'inka a la Madre Tierra en el Apurímac (Valderrama y Escalante, 1976), Apu Qorpuna. Visión del mundo de los muertos en la Comunidad de Awkimarka (Valderrama y Escalante, 1980). El articulo Canciones de imploración y de amor en los Andes (Valderrama y Escalante, 1993) es relativamente inusual, pues no está centrado en los contextos etnográficos de las prácticas mismas sino más bien en las canciones que se ejecutan en cuatro distintos contextos en dos comunidades de la provincia de Cotabambas: canciones para el ganado, para la papa, bailes nocturnos de solteros y otras más bien cotidianas. Un texto que provee descripciones etnográficas de prácticas extendidas en todos los Andes alrededor de las montañas y da una idea de su gran heterogeneidad es Montañas sagradas y rituales en los Andes (Valderrama y Escalante, 2012a). En este capítulo, Ricardo y Carmen presentan material relativo a prácticas en las que participaron en dos contextos cusqueños -Quispicanchis y Cusco-, Caylloma en Arequipa y Cotabambas en Apurímac. Finalmente, estos textos etnográficos también incluyen descripciones y análisis sobre el género y el parentesco en comunidades quechuas. Este es el caso de los artículos Ser mujer, warmi kay. La mujer en la cultura andina (Valderrama y Escalante, 1997b) y Matrimonio en las comunidades quechuas andinas (Valderrama y Escalante, 1998). 
Un cuarto grupo de trabajos está relacionado con el interés de Ricardo y Carmen en la historia. De forma consonante con el grueso de su producción, no sorprende que estén muy interesados en la historia oral y que hayan contribuido a la reflexión sobre este tema en su texto La importancia de la historia oral en Latinoamérica (Valderrama y Escalante, 1988b). Sin embargo, su trabajo histórico no se basa exclusivamente en los testimonios orales sino también en trabajo en distintos tipos de archivos. Uno de sus trabajos históricos es el relativo a un levantamiento indígena en la primera mitad del siglo XX en las provincias de Cotabambas (Apurímac) y Chumbivilcas (Cusco) que justamente teje investigación de archivo con testimonios orales: Levantamiento de los indigenas de Huaquira y Quiñota (1922-1924) (Valderrama y Escalante, 1981). Otra investigación histórica se centra en las prácticas de arrieros en Huancavelica en la primera mitad del siglo XX: Arrieros, troperos y llameros en Huancavelica (Valderrama y Escalante, 1983). Otros textos que pueden ser enmarcados como historias recientes son los textos Desplazados por la violencia política en el Perú: 1980-2000 (Valderrama y Escalante, 2011b) y Balance de las investigaciones en antropología en el Cusco (Valderrama y Escalante, 2008), cuyos títulos son explícitos sobre su contenido. Más recientemente, Ricardo y Carmen se volcaron a la reflexión sobre la historia de las panacas nobles incas reducidas en el pueblo de San Jerónimo de las cuales descienden a través del análisis de documentos de archivos familiares, entre ellos, el suyo propio. Entre estos recientes textos están Ayllus Incas, tierras del Sol y agua del Huanacauri en Sucsu Auccaille, San Jerónimo, Cusco (Escalante y Valderrama, 2020) y Etnicidad y descendencia. Los incas hoy, de carne y hueso: Cusco 2020 (Valderrama y Escalante, 2020).

Si bien este texto no es una revisión exhaustiva de su trabajo, no se pueden dejar de mencionar las tesis de posgrado que Ricardo llevó adelante en los programas de la Pontificia Universidad Católica del Perú y que aún no se han publicado. Estos son la tesis de maestría en Antropología, Sistemas de autoridades en una comunidad quechua contemporánea (Valderrama, 2007) y la tesis de doctorado en Antropología con mención en Estudios Andinos, Pastores, pastos y rebaños en la provincia de Caylloma (Arequipa) (Valderrama, 2012).

No se podrían culminar estas breves palabras sobre la obra de Ricardo (y de Carmen) sin mencionar el rol de su acogedora casa de San Jerónimo en la construcción y mantenimiento de redes entre investigadores locales y extranjeros. A través de sobremesas y largas tertulias con infinidad de anécdotas, su casa ha sido un cálido espacio de conocimiento entre investigadores de diferentes procedencias 
y generaciones, y a través de este, de un aprendizaje permanente y compartido para quienes hemos tenido el privilegio de haber sido acogidos alguna vez en ella.

Ha partido uno de los más notables etnógrafos de las culturas andinas contemporáneas dejándonos una enseñanza radical de compromiso de servicio a la sociedad. Haciendo eco de las palabras de Carmen cuando despedía por última vez a Ricardo en el frontis de la municipalidad cusqueña, repetimos con ella: ¡Haylli, Ricardo Valderrama!

\section{REFERENCIAS BIBLIOGRÁFICAS}

Albó, Xavier. (1973). El futuro de los idiomas oprimidos en los Andes. 9th International Congress of Anthropological and Ethnological Sciences.

Escalante, Carmen y Valderrama, Ricardo. (2020). Ayllus Incas, tierras del Sol y agua del Huanacauri en Sucsu Auccaille, San Jerónimo, Cusco. Anthropologica, 38(45), 161-185. doi.org/10.18800/anthropologica.202002.007

Golte, Jürgen. (1980). Gregorio Condori Mamani o la Bancarrota del Sistema Cognitivo Andino. La Revista, 3, 18-20.

Golte, Jürgen. (1981). ¿Qué es la cultura frente a la historia? La Revista, 4, 59-63.

Gow, Rosalind y Bernabé Condori. (1976). Kay Pacha. Centro Bartolomé de las Casas.

Mannheim, Bruce y Krista Van Vleet. (1998). The dialogics of southern Quechua narrative. American Anthropologist, 100, 326-346.

Nieto, Luis. (2020, agosto 30). En camino hacia el Coropuna. Post de Facebook. Recuperado de https://www.facebook.com/luis.nietodegregori/ posts/10158326615347278

Ossio, Juan M. (1981). Condori Mamani en discusión. La creatividad del pensamiento andino. La Revista, 4, 57-58.

Urbano, Henrique. (1981). Dogmas, bancarrotas y sistemas cognitivos. La Revista, 4, 58-59.

Valderrama, Ricardo. (2007). Sistemas de autoridades en una comunidad quechua contemporánea. Tesis de maestría en Antropología. Pontificia Universidad Católica del Perú.

Valderrama, Ricardo. (2012). Pastores, pastos y rebaños en la provincia de Caylloma (Arequipa). Tesis de doctorado en Antropología. Pontificia Universidad Católica del Perú.

Valderrama, Ricardo. (2015). El inca en la tradición quechua contemporánea. Revista Andina, 53, 277-299. 
Valderrama, Ricardo y Carmen Escalante. (1975). El apu Ausangate en la narrativa popular. Allpanchis Phuturinqa, 8, 175-184.

Valderrama, Ricardo y Carmen Escalante. (1976). Pacha T'inka o la T'inka a la Madre Tierra en el Apurímac. Allpanchis Phuturinqa, 8(9), 177-191.

Valderrama, Ricardo y Carmen Escalante. (1977). Gregorio Condori Mamani. Autobiografía. Centro Bartolomé de las Casas.

Valderrama, Ricardo y Carmen Escalante. (1978). Mitos y leyendas de los quechuas del sur del Perú. Debates en Antropología, 2, 125-135.

Valderrama, Ricardo y Carmen Escalante. (1980). Apu Qorpuna. Visión del mundo de los muertos en la Comunidad de Awkimarka. Debates en Antropología, 5, 233-264.

Valderrama, Ricardo y Carmen Escalante. (1981). Levantamiento de los indígenas de Huaquira y Quiñota (1922-1924, Apurímac, Cusco). Universidad Nacional Mayor de San Marcos, Seminario de Historia Rural Andina.

Valderrama, Ricardo y Carmen Escalante. (1983). Arrieros, troperos y llameros en Huancavelica. Allpanchis, 15(21), 65-88.

Valderrama, Ricardo y Carmen Escalante. (1986). Sistemas de riego y organización social en el valle del Colca. Caso Yanque. Allpanchis, 18(27), 179-202.

Valderrama, Ricardo y Carmen Escalante. (1988a). Del Tata Mallku a la Mama Pacha: Riego, sociedad y ritos en los Andes peruanos. Centro de Estudios y Promoción del Desarrollo.

Valderrama, Ricardo y Carmen Escalante. (1988b). La importancia de la historia oral en Latinoamérica. Revista Universitaria, 137, 229-239.

Valderrama, Ricardo y Carmen Escalante. (1988c). Testimonio de un pongo. Oralidad. Anuario para el rescate de la tradición oral en América Latina, 1, 46-53.

Valderrama, Ricardo y Carmen Escalante. (1990). «Nuestras vidas»: abigeos en Cotabambas. En C. Aguirre y C. F. Walker (eds.), Bandoleros, abigeos y montoneros: criminalidad y violencia en el Perú, siglos XVIII-XX (pp. 309-332). Instituto de Apoyo Agrario.

Valderrama, Ricardo y Carmen Escalante. (1992). Nosotros los humanos: ñuqanchik runakuna: testimonio de los quechuas del siglo XX. CBC.

Valderrama, Ricardo y Carmen Escalante. (1993). Canciones de imploración y de amor en los Andes. Literatura oral de los quechuas del siglo XX. Revista de Crítica Literaria Latinoamericana, 19(37), 11-39. doi.org/10.2307/4530636

Valderrama, Ricardo y Carmen Escalante. (1995). El Inka Vive. Bulletin de la Société Suisse des Américanistes, 59-60, 107-118. 
Valderrama, Ricardo y Carmen Escalante. (eds.). (1996). Andean Lives: Gregorio Condori Mamani and Asunta Quispe Huamán (P. H. Gelles y G. Martínez, trads.). University of Texas Press.

Valderrama, Ricardo y Carmen Escalante. (1997a). La doncella sacrificada: mitos del valle del Colca. Universidad Nacional de San Agustín de Arequipa; Instituto Francés de Estudios Andinos.

Valderrama, Ricardo y Carmen Escalante. (1997b). Ser mujer, warmi kay. La mujer en la cultura andina. En D.Y. Arnold (ed.), Más allá del silencio. Las fronteras de género en los Andes (pp. 153-170). CIASE; ILCA.

Valderrama, Ricardo y Carmen Escalante. (1998). Matrimonio en las comunidades quechuas andinas. En D.Y. Arnold (ed.), Gente de carne y hueso: las tramas de parentesco en los Andes. CIASE: ILCA.

Valderrama, Ricardo y Carmen Escalante. (2000). Agua, riego, alianza y competencia en mitos del Cusco. Senri Ethnological Reports, 18, 261-294. doi.org/ info:doi/10.15021/00002165

Valderrama, Ricardo y Carmen Escalante. (2008). Balance de las Investigaciones en Antropología en el Cusco. En A. Diez (ed.), La antropología ante el Perú de hoy: balances regionales y antropologías latinoamericanas. CISEPA PUCP.

Valderrama, Ricardo y Carmen Escalante. (2011b). Desplazados por la violencia política en el Perú: 1980-2000. En M. Lienhard (ed.), Expulsados, desterrados, desplazados / Expulsos, desterrados, deslocados (pp. 157-169). Vervuert Verlagsgesellschaft.

Valderrama, Ricardo y Carmen Escalante. (2012a). Montañas sagradas y rituales en los Andes. En W. Rozas y D. Valencia (eds.), Cultura andina: Cosmovisión, arqueología (pp. 19-50). Convenio CIUF-UNSAAC.

Valderrama, Ricardo y Carmen Escalante. (2020). Etnicidad y descendencia. Los incas hoy, de carne y hueso: Cusco 2020. Americanía: Revista de Estudios Latinoamericanos, 11, 160-192. 